\title{
Evaluación Remota de Aprendizajes en la Universidad: decisiones docentes para encarar un nuevo desafío
}

\section{(Remote Learning Assessments in Higher Education: Teaching Decision Making when Facing a New Challenge)}

\author{
Gisela Schwartzman \\ Carolina Roni \\ Michelle Berk \\ Erica Delorenzi \\ Mariana Sánchez \\ María Laura Eder \\ Instituto Universitario Hospital Italiano de Buenos Aires, IUHIBA (Argentina)
}

DOI: https://doi.org/10.5944/ried.24.2.29078

\section{Cómo referenciar este artículo:}

Schwartzman, G., Roni, C., Berk, M., Delorenzi, E., Sánchez, M., y Eder, M. L. (2021). Evaluación Remota de Aprendizajes en la Universidad: decisiones docentes para encarar un nuevo desafío. RIED. Revista Iberoamericana de Educación a Distancia, 24(2), pp. 67-85. https://doi.org/10.5944/ried.24.2.29078

\section{Resumen}

La pandemia por COVID-19 y la suspensión de actividades presenciales enfrentó a las universidades con el desafío de remotizar sus funciones. Este trabajo se propone caracterizar propuestas de evaluación de aprendizajes diseñadas, al inicio del confinamiento, por docentes de grado y posgrado de ciencias de la salud en el contexto de una propuesta de Formación para la Virtualización de la Enseñanza (FVE) de un Instituto Universitario. Este estudio descriptivointerpretativo analiza, desde un enfoque cualitativo, 51 planificaciones docentes de evaluación remota elaboradas en el contexto de la formación ofrecida. Las mismas se organizaron en una matriz documental para su categorización e identificación de rasgos prevalentes en estos diseños didácticos con el fin de construir hipótesis interpretativas.

Se evidencian propuestas diversas que mayormente evalúan los aprendizajes con intención de acreditar saberes. Prevalecen diseños de evaluación individual con un uso activo del conocimiento ligado a prácticas académicas y profesionales. Un gran porcentaje prevé su desarrollo en forma asincrónica, mediados por la escritura y con el uso de un amplio repertorio 
de herramientas digitales. Estas propuestas jerarquizan el andamiaje docente a estudiantes durante el proceso de evaluación. Finalmente, se sistematizan los campos emergentes que estructuraron el diseño de las propuestas de evaluación remota de los aprendizajes. Se genera así un insumo para pensar las prácticas evaluativas en el contexto de remotización de la enseñanza. Esperamos que esta contribución trascienda el escenario actual para identificar potencialidades ante la construcción de propuestas híbridas en la post pandemia y en otras disciplinas universitarias.

Palabras clave: formación de profesores; práctica pedagógica; evaluación remota; universidad; tecnología de la educación; COVID-19.

\begin{abstract}
The COVID-19 pandemic and subsequent suspension of on-site academic activity faced universities with the challenge of performing their duties remotely. The study's aim is to characterize learning assessment proposals designed, at the beginning of the lockdown, by graduate and postgraduate health sciences teachers. These proposals were developed in the context of a University Institute teacher-training program on virtualizing education. By using a qualitative approach, this descriptive-interpretative study characterizes 51 remote learning assessment proposals made by teachers taking the course. In order to construct interpretative hypotheses, a matrix for their categorization was created to identify the prevalence of pedagogical traits in the designs.

An array of different proposals were evidenced, most of which were to assess learning with the intention of certifying knowledge. Individual learning assessments prevailed, mostly favoring an active use of knowledge associated with academic and professional practices. A high percentage of teachers planned asynchronous activities by means of writing and the use of a broad array of digital tools. They also prioritized teacher interventions during the assessment process as a scaffolding for the students. Finally, there was a systematization of the emerging characteristics that structured the designs of remote learning assessment proposals. This provides an input toward reflecting on assessment practices in the context of remote teaching. We hope this contribution transcends the current scenario and helps identify potentials for the development of hybrid assessment practices in post pandemic times.
\end{abstract}

Keywords: teacher training education; teaching practice; remote assessments; university; educational technology; COVID-19.

La pandemia por COVID-19 y la suspensión de clases presenciales como medida de cuidado, enfrentó a las universidades al desafío de remotizar ${ }^{1}$ las carreras presenciales. Muchos docentes no contaban con formación en enseñanza virtual, se mostraban resistentes a incluir tecnologías o las incorporaban reforzando prácticas educativas basadas en la transmisión de información. La evaluación instruccional los interpelaba especialmente. ¿Cómo acompañar su tarea en este insólito contexto? ¿Qué previeron al momento de plantear la evaluación remota de los aprendizajes? 
El presente trabajo se desarrolla en un instituto universitario en ciencias de la salud que, a menos de dos semanas de iniciado el confinamiento, desarrolló una estrategia para sostener la continuidad académica incluyendo un plan de Formación Docente para la Virtualización (FVE) para el profesorado de grado, posgrado y sistemas de residencias hospitalarias. Si bien la FVE tuvo continuidad durante todo 2020, en este artículo analizamos datos que se produjeron en su inicio.

La FVE se diferencia de propuestas que capacitan en el uso descontextualizado de herramientas tecnológicas o en la construcción de materiales didácticos digitales exclusivamente (Rapanta et al., 2020). En ella se privilegió el acompañamiento al profesorado para el desarrollo integral de propuestas de educación remota en sus espacios docentes y se propuso dar respuesta a procesos tecnopedagógicos claves ${ }^{2}$, como la evaluación instruccional. Se abordó con un dispositivo de formación que alternó actividades sincrónicas y asincrónicas para el diseño de acciones pedagógicas situadas con una tutoría personalizada (Schwartzman, 2020).

El objeto de este trabajo es caracterizar las propuestas de estos profesores universitarios de ciencias de la salud para la evaluación remota de los aprendizajes, planificadas en el taller de formación durante las primeras semanas de confinamiento por pandemia. Se enfoca la toma de decisiones para evaluar aprendizajes en entornos virtuales en forma remota o híbrida, explorando aspectos como: selección de recursos digitales, instrumentos para evaluar, resultados de aprendizaje a jerarquizar, intervenciones docentes, entre otras.

\section{MARCO TEÓRICO}

Entendemos que la evaluación es parte de un proceso didáctico (Camilloni et al., 1998), inescindible de la enseñanza y el aprendizaje y, por tanto, implica que, por un lado, los estudiantes tomen conciencia de sus aprendizajes y que los docentes interpreten lo que suponen esos aprendizajes construidos, para la enseñanza. Ese proceso didáctico se inicia antes de la clase, en las decisiones, previsiones y supuestos que se ponen en juego para pensar qué y cómo enseñar, y que incluye a la evaluación. Conlleva, por parte del docente, ejercer una mediación entre los estudiantes y determinados saberes. Lo que supone un proceso hacia otros y con otros e involucra un encuentro interpersonal y desde las condiciones reales de partida de los alumnos (Contreras Domingo, 1990).

En este proceso, la evaluación se distingue por el carácter provisorio y complementario de la acción educativa: la respuesta del estudiante es siempre punto de partida para nuevos aprendizajes. De este modo el aprendizaje sólo puede ser acompañado en el sentido longitudinal pues implica construcción y reconstrucción de conocimientos (Hoffmann, 2010). En esta línea, consideramos esencial promover la comprensión y el uso estratégico de lo aprendido para la resolución de problemas y situaciones novedosas por sobre la repetición de ejercicios conocidos (Pozo y Pérez Echeverría, 2009). Del lado de la comprensión podemos encontrar una evaluación 
que reconoce matices y gradualidad en los resultados de aprendizaje; mientras que de la mano de la repetición la evaluación espera respuestas que resultan reproducción de información.

Desde una perspectiva que reconoce la relación multidimensional entre evaluación y aprendizaje pueden identificarse 4 dimensiones (Barberà, 2006) que presentan su complejidad y riqueza y resultan valiosas para abordar los procesos de evaluación tanto presenciales como en línea: evaluación del aprendizaje que acredita ante la sociedad la adquisición de competencias por los estudiantes; evaluación para el aprendizaje basada en la retroalimentación a través del diálogo entre estudiantes y docentes que permite avanzar en el conocimiento; evaluación como aprendizaje que contempla el aprendizaje como parte de la misma dinámica evaluativa; y, evaluación desde el aprendizaje donde se diagnostican conocimientos previos para anclar lo que se enseña.

Para comprender las particularidades de la evaluación remota de los aprendizajes recogemos aportes sobre evaluación en línea para repensarlos en este contexto de emergencia, así como aquellos relativos a la educación en el campo de la salud.

En cuanto a la evaluación en entornos virtuales, identificamos un foco en los instrumentos como los e-portfolio o cuestionarios (Bryan y Clegg, 2019) y en la reutilización de bancos de recursos para hacer más eficiente su administración (Alruwais et al., 2018). Estos abordajes parciales no abordan en profundidad la multidimensionalidad señalada, dejando fuera aspectos críticos como la valoración de los resultados y la toma de decisiones o la comunicación con los estudiantes, por ejemplo. Sin embargo, otros trabajos como el de Quesada Castillo (2006) y Castro (2010) realizan un esfuerzo por conjugar varios componentes de la enseñanza y evaluación en línea, considerando entre éstos los principios, las funciones, los tipos de evaluación, las interacciones y los medios utilizados. Concluyen en la necesidad de usar dicho ordenamiento para orientar el diseño de cursos "en línea".

Concibiendo a la evaluación como parte sustancial de los procesos didácticos, su desarrollo en línea requiere también mirar las prácticas de enseñanza en esa modalidad, considerar los nuevos modos de circulación y construcción social de conocimientos, reconocer las tecnologías digitales disponibles y cómo abonan a la construcción de conocimientos y potencian la modificación de prácticas que requieren ser repensadas. Elena Barberà en 2006 caracterizó lo que supone la inclusión de tecnologías para modificar sustancialmente las prácticas habituales de evaluación instruccional identificando tres aportes: la evaluación automática, enciclopédica y colaborativa. Desde entonces, el desarrollo exponencial de las tecnologías digitales y los nuevos desarrollos teóricos sobre la enseñanza en línea basados en conceptos como el de inclusión genuina de tecnologías (Maggio, 2012) han consolidado nuevas prácticas (Milillo et al., 2019). Propuestas tales como educación transmedia (Odetti et al., 2020), entornos inmersivos (Rose, 2012), la posibilidad de documentar procesos en forma automática (Maggio, 2012), la captura de datos de interacciones en entornos digitales durante las actividades (Di Cerbo, 2020), la construcción de 
objetos con múltiples voces y autorías, la interacción en espacios públicos de la red (wikipedia, redes sociales, etc.), se afilian a estos últimos desarrollos.

Sin embargo, no podemos dejar de señalar que la evaluación en la universidad sigue siendo uno de los procesos más conservadores y esto se profundiza en la modalidad en línea (Dorrego, 2016). Por lo tanto, es esperable que las publicaciones acerca de esta temática estén aún lejos de alternativas innovadoras como las mencionadas. Esto podría deberse, en gran medida, a la fuerte asociación de la evaluación con su función acreditadora y, específicamente en la virtualidad, al temor que suelen manifestar autoridades y docentes asociado a la pérdida de control sobre la identidad de estudiantes y la fiabilidad de aquello que se busca medir (GarcíaPeñalvo, 2020; Harper et al., 2020; D’Souza y Siegfeldt, 2017). Sin desconocer la relevancia de estas cuestiones, entendemos que las prácticas de evaluación educativa, así como las investigaciones sobre las mismas, deben reconocer que éstas son sólo una parte de la problemática y que es posible pensarlas más ampliamente mediante nociones como las evaluaciones auténticas y el reconocimiento del vínculo intrínseco entre enseñanza y evaluación.

En este artículo enfocamos la particularidad de la remotización. Los tiempos de confinamiento han generado nuevas prácticas educativas para responder a la emergencia y los docentes rápidamente debieron construir modos de enseñar y de evaluar de forma remota para asegurar la continuidad académica. En contraste con las experiencias de educación en línea que se planifican para desarrollarse en dicha modalidad, el contexto ha forzado el desarrollo de la Educación Remota de Emergencia (Hodges et al., 2020). Ésta implica la búsqueda inmediata de alternativas para resolver la imposibilidad de acceso presencial a las instituciones. El objetivo primordial no es crear una modalidad con rasgos sólidos sino proporcionar un acceso temporal a la instrucción y rápidamente brindar apoyos pedagógicos que estén disponibles de manera fiable durante la crisis. Son propuestas de educación totalmente en línea para formaciones que, de otro modo, se impartirían cara a cara (o de forma híbrida). Entonces, la enseñanza remota parte del supuesto de que se volverá a la presencialidad una vez que la emergencia haya disminuido. Esta situación impuso que la totalidad de los docentes se sumergieran en la inclusión de tecnologías digitales y que debieran asomarse al territorio en línea para afrontar sus tareas. Esto ha generado un fuerte movimiento en las prácticas universitarias que podría ser oportunidad para promover cambios pedagógicos profundos.

Al relevar publicaciones surgidas a partir de la pandemia por Covid-19 encontramos una primera etapa con manuales o guías (Abella García et al., 2020; Rivera Mata, 2020; University of Twente, 2020) y trabajos que se centran en reconocer barreras para el diseño de evaluación remota tales como: la búsqueda de reproducir en línea lo que se había definido para la presencialidad, la expectativa de retorno a la actividad presencial, las brechas de acceso y conectividad, las dudas sobre la seguridad y autoría de quien se examina, (García-Peñalvo, 2020), el fraude (Harper et al., 2020) y cómo evitarlo mediante el uso de sistemas de supervisión electrónica 
(González-González et al., 2020). En relación con esto último, como mencionamos se trata de preocupaciones habituales de la evaluación en línea que se actualizan en artículos vinculados a la evaluación remota. Por otra parte, algunos estudios avanzan en recomendaciones didácticas que implican la evaluación por competencias en forma remota (García-Peñalvo, 2020) o la mirada sobre los procesos de aprendizaje más que sus resultados (Condori Gutiérrez, 2020; Fardoun et al., 2020).

Habiendo conceptualizado la evaluación en línea y remota, enfocamos consideraciones respecto de la evaluación en disciplinas de las ciencias de la salud. Interesa mencionar un estudio donde se relevaron las preocupaciones sobre evaluación de profesores de este campo durante su formación en docencia universitaria (Schwartzman et al., 2019). En líneas generales, sus preocupaciones giran en torno a la objetividad, una asociación inicial fuerte con la calificación y al interés en los exámenes de selección múltiple o "el oral" como principales instrumentos para reconocer el dominio de conocimientos declarativos. Al transitar una formación pedagógica estas preocupaciones iniciales se transforman en reflexión sobre instrumentos de evaluación adecuados para reconocer aprendizajes y acreditarlos, elaboración de programas de evaluación con instrumentos diversos y decisiones consistentes con la planificación didáctica, revisión de las funciones de la evaluación y su sentido junto con la preocupación por generar situaciones de devolución constructiva de los exámenes como instancias de aprendizaje para los estudiantes, con foco en la especificidad disciplinar al reflexionar sobre el feedback como proceso asociado a la evaluación del desempeño de los profesionales de la salud en contextos clínico-asistenciales.

En otras publicaciones del campo de la educación médica se realizan algunas predicciones sobre los desafíos de la evaluación remota, tales como baja motivación de alumnos (ParkJiao y Lissitz, 2020) y necesidad de potenciar y recuperar información a partir de interacciones de los estudiantes con entornos digitales (Di Cerbo, 2020). También presentan prescripciones por ejemplo para realizar exámenes a libro abierto con el fin de garantizar la validez, confiabilidad y equidad (Er et al., 2020).

En suma, enfrentamos el desafío de evitar en la universidad una evaluación remota conservadora que privilegia la calificación y control de identidad de los estudiantes. El aporte de la presente investigación será sistematizar las propuestas de evaluación remota de profesores de ciencias de la salud que persiguieron una inclusión genuina de tecnologías coherente con los enfoques de enseñanza en los que abrevan. Esperamos que las contribuciones de esta publicación trasciendan este contexto puntual y disciplinar para potenciar la construcción de propuestas híbridas en la post pandemia.

\section{METODOLOGÍA}

En este trabajo se caracterizan propuestas de remotización de actividades de evaluación diseñadas por profesores de ciencias de la salud de grado y posgrado, en 
el contexto de una formación docente para la virtualización de emergencia. Si bien se solicitó la planificación de actividades de evaluación que fueran a concretarse, en este artículo se analiza el diseño instruccional de estas actividades y no su implementación. Estas planificaciones no fueron construidas desde el sentido común sino sustentadas, en alguna medida, por el conocimiento didáctico que implicó la formación docente.

Se planteó un estudio descriptivo-interpretativo, desde un enfoque cualitativo, de 51 diseños de propuestas de evaluación de los aprendizajes que los docentes publicaron en una base de datos de Moodle durante un taller de una semana de duración. Los campos de esta base de datos que organizaron sus diseños fueron: propósito de la evaluación, naturaleza del conocimiento a evaluar, instrumento/s de evaluación, intervenciones previstas del docente, y criterios de valoración de lo realizado por los estudiantes. Para analizar las producciones se construyó una matriz documental.

Se desarrolló un análisis categorizador con el propósito de identificar los rasgos centrales de las planificaciones tomando en conjunto las respuestas de los docentes al interior de cada uno de los campos. Así se categorizaron las respuestas agrupándolas por similitud. Este análisis nos permitió identificar la prevalencia de algunos rasgos de las actividades evaluativas planificadas. En segundo lugar, y con el objetivo de interpretar con mayor profundidad las características de las actividades propuestas, pusimos en relación los resultados encontrados en los campos, lo que nos permitió construir hipótesis interpretativas que compartimos en el siguiente apartado de análisis.

\section{RESULTADOS}

Dado que esta investigación y la acción de formación que es su punto de partida se desarrollaron en el contexto de pandemia por COVID-19, consideramos necesario puntualizar algunas cuestiones que dan sentido a los resultados de la misma.

Como se mencionó, el taller de formación sobre evaluación remota se realizó al inicio del confinamiento y con la hipótesis (luego descartada) de un pronto retorno a la presencialidad. En este sentido, la FVE proponía construir criterios para la selección de contenidos a virtualizar y diferenciar aquellos que se posponían para el regreso a las aulas. En muchos casos, los conocimientos que implicaban la inmersión en prácticas reales fueron inmediatamente postergados para esa instancia. Además, todo lo producido tenía la impronta de resolver los problemas de la continuidad académica en forma urgente e inmediata.

Del análisis emergen dos tipos de resultados. En primer lugar, del procesamiento de los datos matrizados surgen cuatro dimensiones que permiten describir las evaluaciones remotas que planificaron los docentes participantes del taller de formación: 1- propósitos identificados para las evaluaciones, 2- decisiones sobre los instrumentos, 3 - resultados de aprendizaje pasibles de evaluación en el contexto 
remoto, 4- características anticipadas de la intervención docente. En segundo lugar, aportamos una sistematización de los campos que estructuraron los diseños estudiados (y sus configuraciones posibles) que emerge de los datos.

\section{Sobre los propósitos de la evaluación}

La casi totalidad de los docentes diseñaron propuestas vinculadas a la evaluación del aprendizaje (92\%). Encontramos pocos ejemplos donde inferimos otras dimensiones de la evaluación (Barberà, 2006) como evaluación desde el aprendizaje, donde se explicita el interés de conocer los saberes previos de sus estudiantes y cómo pueden ponerlos en juego para resolver situaciones nuevas (10\%). Algunos docentes integran en sus propuestas la evaluación para el aprendizaje, con foco en el feedback con el objeto de que los alumnos evalúen cómo va su propio aprendizaje y brindando información que los ayude a seguir pensando y produciendo. Por último, otros docentes señalan que la información recogida en la evaluación será un insumo para valorar cómo está resultando la cursada (15\%).

Interpretamos que la alta frecuencia de propuestas de evaluación de los aprendizajes probablemente es producto de la preocupación por responder a una tarea primordial de la universidad, esto es calificar para acreditar saberes, exigencia que recae sobre los docentes responsables de las actividades curriculares. Ante la incertidumbre generada por la pandemia es posible que hayan aprovechado el contexto de formación, con tutoría, para privilegiar decisiones en este sentido.

\section{Sobre los instrumentos de evaluación}

Respecto delosinstrumentos de evaluación previstos en sus diseños, identificamos características particulares respecto a cómo se desarrollan temporalmente, qué gestión de la grupalidad plantean, cuáles son las prácticas de estudio que involucran, qué tipo de producto es esperado, cuáles son los recursos digitales seleccionados y los criterios de valoración privilegiados.

En primer lugar, respecto del desarrollo temporal, encontramos que más de la mitad de los docentes optó por la asincronía (55\%) y casi un $25 \%$ por una modalidad mixta donde se combinan instancias de trabajo asincrónico y sincrónico. Si bien estas actividades se asemejan a los exámenes domiciliarios, se desarrollan en una temporalidad regulada por el docente junto a los estudiantes. Esto rompe con la tradicional forma de evaluar en la presencialidad en un tiempo limitado de aula y permite a los estudiantes gestionar los momentos de resolución de la actividad en forma más prolongada. Así, encontramos una adaptación y no una copia de la presencialidad.

En relación con la gestión de la grupalidad, la mayoría optó por evaluaciones individuales (61\%), y algunos por evaluaciones grupales (16\%). Cabe destacar que también se diseñaron propuestas con etapas combinando momentos de evaluación 
individual, de a pares o grupos (14\%). Un 9\% no explicita el agrupamiento. Nos preguntamos si la preeminencia de lo individual se vincula con una mayor seguridad de los docentes respecto de qué aprendizajes atribuir a cada estudiante para calificarlos. Evaluar una producción grupal resulta más desafiante, en tanto la individual es una respuesta más convencional para cumplir con el requerimiento de toda institución universitaria de calificar.

Dentro de las prácticas de estudio involucradas en las evaluaciones, la escritura es la que presenta mayor porcentaje (80\%), aunque, suele conjugarse con la lectura, oralidad y/o demostración audiovisual.

Asimismo, encontramos que el tipo de actividad esperada por parte del estudiante remite, en ciertas ocasiones, a prácticas propias del perfil profesional para el que forman y en otras a prácticas inherentes a la vida universitaria vinculadas con el estudiantar (Fenstermacher, 1989). Resulta interesante que se privilegien actividades pertenecientes al primer grupo como análisis de casos clínicos (35\%), resolución de ejercicios que recrean prácticas asistenciales (14\%), demostración de maniobras (10\%), redacción de informes de laboratorio (6\%) y resolución de problemas profesionales habituales (6\%). Aunque también encontramos producciones propias del estudiante universitario como resolución de cuestionarios sobre conocimientos declarativos (20\%), escritos monográficos y de reflexión argumental (18\%), exposiciones orales sobre lo estudiado (12\%) y armado de producciones gráficas que reorganizan el conocimiento disciplinar (4\%).

Finalmente, los recursos digitales escogidos para vehiculizar estas evaluaciones fueron diversos: herramientas de videoconferencia (20\%), gestor de archivos del campus virtual institucional (16\%), documentos colaborativos (14\%), videos (14\%), foros (12\%), cuestionarios o formularios (10\%), correo electrónico (4\%) y simuladores virtuales (2\%). Sin embargo, resulta llamativo que muchos docentes no especificaron este dato (25\%) al diseñar su propuesta.

Para estas evaluaciones los docentes propusieron criterios de valoración diversos. Encontramos una alta presencia de aquellos vinculados a que los estudiantes puedan jerarquizar, sintetizar, integrar, fundamentar conceptos, etc. También incluyeron criterios vinculados con el pensamiento crítico como analizar situaciones, problematizar o realizar búsqueda bibliográfica crítica. Debido a la preponderancia de producciones escritas, se propusieron valorar aspectos formales como uso de vocabulario disciplinar, claridad de la redacción y formatos de entrega.

\section{Sobre los resultados de aprendizaje a evaluar}

Los resultados de aprendizaje que los docentes se propusieron evaluar, probablemente fueron aquellos que en los inicios de la cuarentena consideraron posibles y viables. Ya se ha mencionado que la hipótesis era de pronto retorno a la presencialidad. Identificamos dos tipos de resultados de aprendizaje buscados: 1. aprendizajes repetitivos y 2. aprendizajes vinculados a la comprensión de las 
prácticas profesionales: A. para su análisis y B. para su ejercicio. A continuación, explicamos e ilustramos esta categorización con fragmentos textuales de la empiria.

1. Aprendizajes enfocados en la repetición de ciertos datos, hechos, estructuras. Encontramos sólo 4 casos (8\%) en esta categoría. Algunas de las actividades que ilustran este tipo de evaluación son: "Completar las partes en un gráfico"; "Señalar estructuras anatómicas."

2. Aprendizajes enfocados en la comprensión y la resolución de problemas. Se trata de conocimientos vinculados a las prácticas profesionales ya sea para analizarlas o para recrearlas. Presentamos ambas variantes:

A. Análisis de prácticas profesionales: acá se agrupan la mayor cantidad de propuestas (55\%), con variadas intenciones como "Que sean capaces de identificar conductas/factores de evolución..."; "Que sean capaces de reflexionar sobre los determinantes sociales de la salud, contextualizar las intervenciones y entender la salud como un derecho". En estos casos las actividades que vehiculizan la evaluación son, por ejemplo "el análisis de situaciones desde aspectos éticos"; "la lectura crítica; "la interpretación de gráficos de fenómenos reales"; "el desarrollo de una justificación completa de todas las respuestas ofrecidas frente a una situación-problema".

B. Recreación de las prácticas profesionales. Encontramos un número considerable (37\%) de este tipo de evaluaciones centradas en dos tipos de prácticas disciplinares: vinculadas a la actividad médico-asistencial y a la actividad académica (Villavicencio, 2018). Inferimos aquí el esfuerzo docente de preservar en la virtualidad la formación y evaluación de resultados de aprendizaje estrechamente vinculados a la práctica profesional, con aprendizajes situados y sosteniendo la complejidad de aprender el "juego completo" (Perkins, 2010). Se trata de actividades desafiantes (problemas, casos demostraciones o simulaciones realizadas por los estudiantes) que requieren la toma de decisiones. Los casos salientes, acordes con esas intenciones, son por ejemplo el desarrollo de una formulación farmacológica (se les da el medicamento y "deben decidir con qué componentes lo van a formular, seleccionar los excipientes y envase a utilizar"); entrevistar pacientes mediante role playing ("filmar un video entre pares donde uno tendrá el rol de médico y otro de familiar del paciente-niño"); la resolución de un caso complejo, ("respondan cómo lo harían, describiendo pasos y maniobras a realizar"); o comunicarse con un paciente ficticio ("escribirle a un paciente").

Vemos entonces que los resultados de aprendizajes buscados se relacionan con las tareas propuestas y su complejidad. La búsqueda de resultados vinculados a la repetición plantea tareas de menor dificultad y, al promover la comprensión se 
plantean, en cambio, actividades de mayor complejidad para el estudiante. Dicho desafío promueve aprendizajes más duraderos y transferibles a la práctica profesional para la cual se están formando (Pozo y Pérez Echeverría, 2009).

\section{Sobre las intervenciones del docente}

Analizamos los modos de intervenir estructuradas en 3 aspectos: momentos y tipos de intervención, razones y vías para hacerlo y diferentes modalidades de devolución.

Cuando analizamos los momentos en que intervienen, la mayor parte de las producciones (55\%) prevé la intervención docente durante el proceso tendientes a aclarar dudas u orientar la resolución de la tarea. Otras respuestas (26,5\%) refieren exclusivamente a los "extremos" (Carlino et al., 2013), es decir al inicio o cierre de la instancia de evaluación (incluyendo docentes que intervienen en ambos extremos o sólo en uno de ellos). También hay quienes se plantean hacerlo de forma combinada en los extremos y durante toda la evaluación (18,5\%). Al encontrarnos con la gran cantidad de menciones sobre las intervenciones durante el proceso, nos preguntamos si será este uno de los aprendizajes que surgen del taller. O será por la "distancia" que la situación impuso y la necesidad de fortalecer vínculos pedagógicos.

Las acciones que configuran las intervenciones varían según el momento en que se realizan. Cuando refieren al inicio, por ejemplo, la intervención está vinculada a la presentación o explicación de la consigna. Durante el proceso mencionan acciones diversas como responder dudas, formular preguntas desafiantes, sugerir fuentes de información, acompañar, estimular y guiar, organizar el plenario, aclarar conceptos. $\mathrm{Al}$ final se orientan a dar devoluciones. El fragmento que sigue es un ejemplo del tipo de intervenciones que se propone realizar un docente durante la evaluación: "El docente intervendrá durante el proceso de producción dando una devolución constructiva a las producciones ayudando a seguir pensando y poder llegar al encuentro sincrónico con material trabajado".

Las intervenciones difieren además en tanto hay docentes que prevén hacerlo cuando los estudiantes lo soliciten "para contestar dudas", "por si no entienden las consignas" mientras otros consideran valioso y adecuado actuar sin esperar la demanda de sus estudiantes "el docente realizará una intervención de proceso, realizando un acompañamiento al estudiante en la producción individual, orientando dicha producción, redirigiendo a la bibliografía o a un proceso reflexivo, dependiendo del recorrido del estudiante".

Respecto de las vías y herramientas que utilizan los docentes para comunicarse con sus estudiantes e intervenir, el espacio más mencionado es el de los foros (27\%). Este es utilizado como "tablón de anuncios" para comunicar unidireccionalmente consignas y no tanto para habilitar el intercambio y la construcción colectiva de respuestas e interpretaciones. El resto de las menciones refieren a intervenciones mediante correo electrónico (14\%), comentarios al margen en documentos 
compartidos en línea (14\%) y, en menor medida, a las consultas en encuentros sincrónicos (8\%) o videollamadas (6\%). Los últimos dos casos responden a solicitudes de los estudiantes a diferencia de lo ya planificado que se vehiculiza por las primeras vías identificadas.

Por último, destacamos que quienes diseñan intervenciones al final prevén mayormente el feedback individual. Sin embargo, algunos docentes planifican realizar además un plenario para recuperar las respuestas más adecuadas, las dificultades más comunes, tal como ilustran los siguientes fragmentos: "Al recibir las producciones se realizará un feedback general sobre los puntos en común e individual sobre cada trabajo presentado."; "Una vez corregidos los exámenes la docente hará un cierre donde sistematizará el trabajo realizado por el grupo".

La descripción realizada revela la multiplicidad de aristas que desafían la tarea docente en la evaluación remota y da cuenta de la gran cantidad de decisiones que deben tomarse y que van más allá de la elección de un instrumento y del resultado de aprendizaje a evaluar. A continuación, proponemos una organización sistemática de estos datos.

\section{Sistematización de los campos emergentes que estructuraron el diseño de las propuestas de evaluación remota de los aprendizajes}

Se muestra a continuación una síntesis del análisis presentado que sistematiza los campos emergentes y las diversas configuraciones adoptadas. Estos campos y sus configuraciones organizados en la figura 1 muestran en qué se concentraron las decisiones de los docentes en la tarea de remotizar la evaluación de los aprendizajes.

En subsiguientes etapas de este estudio esta sistematización podría someterse a una revisión crítica y justificación a la luz de los marcos teóricos y contribuir en la construcción y validación de una guía para el diseño de la evaluación virtual. Sin embargo, ambos objetivos exceden la extensión de esta publicación. 


\section{Figura 1}

Campos emergentes que estructuraron el diseño instruccional de propuesta de evaluación remota de los aprendizajes en ciencias de la salud

\begin{tabular}{|c|c|c|c|c|}
\hline \multicolumn{5}{|c|}{$\begin{array}{c}\text { Campos emergentes que estructuraron el diseño instruccional de propuestas de } \\
\text { evaluación remota de los aprendizajes en ciencias de la salud }\end{array}$} \\
\hline \multicolumn{5}{|c|}{$\begin{array}{l}\text { A continuación se presenta una sistematización de los campos que emergen de los diseños de evaluación } \\
\text { remota de los aprendizajes llevados adelante por docentes que participaron de este estudio. Los campos, y } \\
\text { configuraciones adoptadas, no son excluyentes ni generalizables a toda propuesta de evaluacion remota de } \\
\text { los aprendizajes. }\end{array}$} \\
\hline \multicolumn{5}{|c|}{ Sobre la consigna de evaluación } \\
\hline \multicolumn{5}{|l|}{ 1. Tipo de actividad: } \\
\hline Análisis de casos & \multicolumn{2}{|c|}{ Resolución de Problemas } & \multicolumn{2}{|c|}{ Resolución de ejercicios } \\
\hline Resolución de cuestionarios & \multicolumn{2}{|c|}{ Producción de informes } & \multicolumn{2}{|c|}{ Elaboración de Monografías } \\
\hline Exposición oral & \multicolumn{2}{|c|}{ Demostración de habilidades } & \multicolumn{2}{|c|}{ Confección de productos gráficos } \\
\hline \multicolumn{5}{|l|}{ 2. Prácticas involucradas: } \\
\hline Lectura & Dralidad & \multicolumn{2}{|c|}{ Escritura } & $\begin{array}{c}\text { Demostración } \\
\text { audiovisual }\end{array}$ \\
\hline \multicolumn{5}{|l|}{ 3. Resultados de aprendizaje: } \\
\hline \multirow{2}{*}{\multicolumn{2}{|c|}{ Repetición }} & \multicolumn{3}{|c|}{ Comprensión } \\
\hline & & \multicolumn{2}{|c|}{ Prácticas profesionales } & Prácticas académicas \\
\hline \multicolumn{5}{|c|}{ 4. Criterios de valoración (de las producciones de los estudiantes): } \\
\hline Integración & \multicolumn{2}{|c|}{ Sintesis } & \multicolumn{2}{|c|}{ Jerarquización de ideas } \\
\hline Fundamentación conceptual & \multicolumn{2}{|c|}{$\begin{array}{l}\text { Identificación/ Ejemplificación de } \\
\text { conceptos }\end{array}$} & \multicolumn{2}{|c|}{ Análisis de una situación } \\
\hline Búsqueda bibliográfica crítica & \multicolumn{2}{|c|}{ Problematización conceptual } & \multicolumn{2}{|c|}{ Inclusión de lenguaje disciplinar } \\
\hline \multicolumn{2}{|c|}{ Claridad de redacción/ presentación de ideas } & Plaz & $y$ form & s de entrega \\
\hline & bre el procec & nto de evaluaci & & \\
\hline 1. Gestión del tiempo: & & & & \\
\hline Sincrónico & & & Asin & nico \\
\hline 2. Grupalidad: & & & & \\
\hline Individual & Gru & equeño & & Grupo-clase \\
\hline 4. Herramienta digital a utilizar: & & & & \\
\hline Herramienta de videoconferencia & Documento & orativo en linea & Cuest & ario/ formulario en línea \\
\hline Foro de intercambio & dlador virtual & Documento & jital & Video \\
\hline & Sobre la int & nción docente & & \\
\hline 1. Momento: & & & & \\
\hline Inicio del proceso & Durar & proceso & & Final del proceso \\
\hline 2. Vías de comunicación: & & & & \\
\hline Correo electrónic & & & ro de it & rcambio \\
\hline Encuentro por videocon & rencia & Comentari & en doc & nento colaborativo \\
\hline 3. Objetivo: & & & & \\
\hline Presentar/ explicar la consigna & Moderar un & cambio grupal & Form & ir preguntas desafiantes \\
\hline Aclarar conceptos & Resp & r dudas & & Dar devolución \\
\hline Andamiar el proce & & Suge & fuente & e infromación \\
\hline 4. Destinatarios: & & & & \\
\hline Cada estudiante & Gru & equeño & & Grupo-clase \\
\hline
\end{tabular}




\section{CONCLUSIONES}

Del análisis de los datos podemos extraer las siguientes conclusiones:

1. La mayoría de los docentes centró su propuesta en la evaluación de los aprendizajes. Como se mencionó anteriormente, es probable que la necesidad de acreditar saberes tenga que ver con esta decisión.

2. Privilegiaron el diseño de evaluaciones asincrónicas vinculadas con la producción escrita individual. Entendemos que la escritura es privilegiada porque permite reconstruir huellas de los aprendizajes y gestionar un tiempo de menor premura al estudiante y al docente que valorará su actividad. Entonces, ante la novedad del contexto impuesto, el producto "texto", como práctica habitual compartida entre docentes y estudiantes universitarios, y la dimensión tiempo, se convirtieron en aliados para gestionar el desafío. Aunque también fueron consideradas instancias grupales, sincrónicas y de discusión oral.

3. Preservaron la naturaleza y especificidad del contenido de enseñanza de las ciencias de la salud al momento de diseñar las actividades evaluativas. En sus propuestas jerarquizaron las prácticas complejas y competencias del ejercicio profesional. Pese a la dificultad de concurrir a escenarios reales de práctica propios del campo de la salud, gran parte de los docentes apostaron a la comprensión a través de evaluaciones ligadas al análisis y recreación de prácticas profesionales.

4. Para esto recurrieron a un repertorio de recursos digitales diversos. Esto da cuenta de cierta apropiación tecnológica y los esfuerzos de docentes por privilegiar la naturaleza de lo que querían evaluar, yendo más allá del mandato de acreditar aprendizajes que podría resolverse con un cuestionario sincrónico.

5. En relación a las intervenciones docentes previstas, la mayoría optó por hacerlo durante el proceso para andamiar a sus estudiantes. La vía más elegida para las comunicaciones fue el foro.

\section{REFLEXIONES FINALES}

El confinamiento que implicó el COVID-19 enfrentó a los docentes al doble desafío de remotizar la enseñanza y la evaluación. En la urgencia inicial, y con la hipótesis subyacente de un pronto retorno a la presencialidad, encontramos un repertorio variado de propuestas de evaluación diseñadas por docentes universitarios en ciencias de la salud. Éstas se constituyen en un punto de partida valioso para pensar con más profundidad el campo de posibilidades que ofrece la remotización de la enseñanza y la evaluación.

Los docentes se vieron en la necesidad de revisar sus decisiones pedagógicas y encarar la tarea de evaluar evitando trasladar las prácticas presenciales a la virtualidad. En este sentido, fueron desafiados a concebir a la evaluación como parte sustancial de los procesos didácticos, y su desarrollo en línea les requirió mirar 
integralmente las prácticas de enseñanza en esa modalidad, considerar los nuevos modos de circulación en red de conocimientos plasmados en múltiples lenguajes, reconocer las tecnologías digitales disponibles y cómo abonan a la construcción de conocimientos al mismo tiempo que potencian la modificación de prácticas académicas que requieren ser repensadas.

Entre los resultados más salientes aportamos qué rol asumen los docentes durante el desarrollo de las actividades de evaluación. Este foco es escasamente abordado por la literatura sobre evaluación, a excepción de los estudios que adoptan miradas críticas sobre el uso (y abuso) del poder en la toma de exámenes o sobre cómo hacer devoluciones. Comprender la evaluación como parte del proceso didáctico supone pensar entonces cómo interviene el docente para sostener (y comprobar) los procesos de aprendizaje de sus estudiantes

En este estudio nos planteamos la necesidad y el valor de recuperar las preguntas y decisiones que formularon los docentes para promover mejores prácticas de enseñanza y evaluación. Desde esta visión sistematizamos los campos emergentes que estructuraron el diseño de las propuestas de evaluación remota de los aprendizajes.

Creemos que muchas respuestas surgirán de nuevas investigaciones y de programas que, como los talleres de la FVE, continúen abordando la formación como invitación a la construcción colectiva y reflexión compartida e incorporen otras miradas para formar docentes reflexivos de sus prácticas educativas en línea, presenciales e híbridas.

\section{AGRADECIMIENTO}

Agradecemos la colaboración de la becaria Katherine Korniejczuk en la búsqueda y sistematización de antecedentes y la revisión atenta de este manuscrito a la Dra. Natalia Rosli.

\section{NOTAS}

1. Entendemos por remotizar a las acciones para sostener la actividad académica originalmente presencial en contextos de emergencia.

2. El Plan de Formación para la Virtualización de la Enseñanza se desarrolló durante todo el ciclo académico 2020 en torno a: a) procesos tecno-pedagógicos, b) autonomía docente en la gestión de recursos tecnológicos, c) programación de la enseñanza, d) participación de estudiantes en la sincronía.

\section{REFERENCIAS}

Abella García, V., Grande de Prado, M., García-Peñalvo, F. J., y Corell Almuzara, A. (2020). Guía de recomendaciones para la evaluación online en las Universidades Públicas de Castilla y León (Versión 
1.1). Zenodo. http://doi.org/10.5281/ zenodo.3780661

Alruwais, N., Wills, G., y Wald, M. (2018). Advantages and challenges of using e-assessment. International Journal of Information and Education Technology, 8(1), 34-37. https://doi.org/10.18178/ ijiet.2018.8.1.1008

Barberà, E. (2006). Aportaciones de la tecnología a la e-Evaluación. RED. Revista de Educación a Distancia, 5(6), 1-13. https://epk.is/11WZq

Bryan, C., y Clegg, K. (Eds.). (2019). Innovative assessment in higher education: A handbook for academic practitioners. Routledge. https://doi. org/10.4324/9780429506857

Camilloni, A., Celman, S., Litwin, E., y Palou de Maté, M. D. C. (1998). La evaluación de los aprendizajes en el debate didáctico contemporáneo. Paidós.

Carlino, P., Iglesia P., Bottinelli, L., Cartolari, M., Laxalt, I. y Marucco, M. (2013). Leer y escribir para aprender en las diversas carreras y asignaturas de los IFD que forman a profesores de enseñanza media: concepciones y prácticas declaradas de los formadores de docentes. Buenos Aires: Ministerio de Educación de la Nación. https://www.aacademica.org/paula. carlino/141

Castro, E. P. (2010). Evaluación de los aprendizajes y estudio de la interactividad en entornos en línea: un modelo para la investigación. RIED. Revista Iberoamericana de Educación a Distancia, 13(1), 17-38. https://doi. org/10.5944/ried.1.13.886

Condori Gutiérrez, J. (2020). La evaluación de las competencias en un modelo educativo emergente. Aportes desde el enfoque socioformativo. En J. LunaNemecio (Coord.), Memorias del Quinto Congreso Internacional de Evaluación Socioformativa (VALORA-2O2O). Congreso conducido por el Centro
Universitario CIFE de Cuernavaca, México. https://cife.edu.mx/recursos

Contreras Domingo, J. (1990). Enseñanza, curriculum y profesorado. Introducción crítica a la didáctica. Akal.

Di Cerbo, K. (2020). Assessment for Learning with Diverse Learners in a Digital World. Educational Measurement: Issues and Practice, 39, 90-93. https://doi. org/10.1111/emip.12374

Dorrego, E. (2016). Educación a Distancia y Evaluación del Aprendizaje. RED. Revista de Educación a Distancia.5O(12). https:// doi.org/10.6018/red/50/12

D’Souza, K. A., y Siegfeldt, D. V. (2017). A conceptual framework for detecting cheating in online and take-home exams. Decision Sciences Journal of Innovative Education, 15(4), 370-391. https://doi. org/10.1111/dsji.12140

Er, H., Nadarajah, V., Wong, P., Mitra, N. (2020). Practical Considerations for Online Open Book Examinations in Remote Settings. MedEdPublish, 9(1),153. $\quad$ https://doi.org/10.15694/ mep.2020.000153.1

Fardoun, H., González, C., Collazos, C., y Yousef, M. (2020). Estudio exploratorio en Iberoamérica sobre procesos de enseñanza-aprendizaje y propuesta de evaluación en tiempos de pandemia. Education in the Knowledge Society. https://epk.is/i5VIk

Fenstermacher, G. (1989). Tres aspectos de la filosofía de la investigación sobre la enseñanza. En M. Wittrock (Comp.), $L a$ investigación en la enseñanza Tomo 1. Paidós, M.E.C.

García-Peñalvo, F. J. (2020). Evaluación online: la tormenta perfecta. Ensinar $A$ Distância. https://repositorio.grial.eu/ bitstream/grial/2007/1/Tormenta.pdf

González-González, C., Infante Moro, A., e Infante Moro, J. C. (2020). Implementation of E-proctoring in Online Teaching: A Study About Motivational 
Factors. Sustainability. 1, 1-13. https:// doi.org/10.3390/su12083488

Harper, R., Bretag, T., Rundle, K. (2020). Detecting contract cheating: examining the role of assessment type. Higher Education Research \& Development, 39, 1-16. https://doi.org/10.1080/07294360. 2020.1724899

Hodges, C., Moore, S., Lockee, B., Trust, T., y Bond, A. (2020). The difference between emergency remote teaching and online learning. Educause Review, 27. https:// er.educause.edu/articles/2020/3/thedifference-between-emergency-remoteteaching-and-online-learning

Hoffmann, J. (2010). Evaluación mediadora. Una propuesta fundamentada. En R. Anijovich (Comp.), La Evaluación significativa, 73-102.

Maggio, M. (2012) Enriquecer la Enseñanza. Los ambientes con alta disposición tecnológica como oportunidad. Paidós.

Milillo, C., Odetti, V., Rogovsky, C., y Trech, M. (2019). Formatos innovadores para propuestas de educación en línea. En G. I. El Jaber, (Comp.), Actas de IV Jornadas Educación a distancia y Universidad, Ciudad Autónoma de Buenos Aires: Flacso Argentina, 2020. http://www. pent.org.ar/institucional/publicaciones/ formatos-innovadores-para-propuestaseducacion-linea

Odetti, V., Schwartzman, G., y Bosch, M. E. (2020). Formatos innovadores para propuestas de educación en línea: una experiencia transmedia y gamificada. Boletín SIED, 1(1), 66-72. https://revista. sied.mdp.edu.ar/index.php/boletin/ article/view/25

ParkJiao, H., y Lissitz, R. W. (2020). What Hath the Coronavirus Brought to Assessment? Unprecedented Challenges in Educational Assessment in 2020 and Years to Come. Educational Measurement: Issues and Practice, 39, 45-48. https://doi.org/10.1111/ emip. 12363
Perkins, D. (2010). El aprendizaje pleno. Principios de la enseñanza para transformar la educación. Paidós.

Pozo, J. I., y Pérez Echeverría, M. P. (2009). Psicología del aprendizaje universitario: La formación en competencias. Morata.

Quesada Castillo, R. (2006). Evaluación del aprendizaje en la educación a distancia "en línea". RED. Revista de Educación a Distancia, M6 (Número especial dedicado a la evaluación en entornos virtuales de aprendizaje). http://www.um.es/ead/ red/M6

Rapanta, C., Botturi, L., Goodyear, P., Guàrdia, L., y Koole, M. (2020). Online university teaching during and after the Covid-19 crisis: Refocusing teacher presence and learning activity. Postdigital Science and Education, 1-23. https://doi. org/10.1007/s42438-020-00155-y

Rivera Mata, J. (2020). How to Teach Online? Recommendations for the assessment of online exams with University students in the USA in times of pandemic. IJERI: International Journal of Educational Research and Innovation, 15, 188-202. https://doi.org/10.46661/ijeri.5003

Rose, F. (2012). The art of immersion: how the digital generation is remaking. Hollywood, Madison Avenue, and the way we tell stories. W. W. Norton \& Company Editor.

Schwartzman, G. (2020). Formación docente para "virtualizar de emergencia": aportes a la respuesta universitaria ante la pandemia COVID-19. Revista Hospital Italiano de Buenos Aires, 40(1), 49-51. https://epk.is/fqoXd

Schwartzman, G., Roni, C., Sánchez, M., Delorenzi, E., y Eder, L. (2019). Perspectiva académica de la enseñanza del profesorado universitario: ¿̇sobre qué prácticas reflexionan los docentes a partir de su formación? Congreso Europeo de Scholarship of Teaching and Learning (EuroSoTL). https://epk.is/qVMbq 
University of Twente (2020). Choosing remote assessment method \& tool. TELT. Technology Enhanced Learning \& Teaching. https://tinly.co/MtkHg
Villavicencio, M. (2018). Comunicación académica: prácticas de lectura y escritura en el aula. Don Bosco.

\section{PERFIL ACADÉMICO Y PROFESIONAL DE LOS AUTORES}

Gisela Schwartzman. Mg. en Enseñanza y Aprendizaje Abiertos y a Distancia (UNED, España). Lic. en Ciencias de la Educación (UBA, Argentina). Coordinadora de Docencia Universitaria y directora de la Especialización en Docencia universitaria IUHIBA. Coordinadora Pedagógica PENT-FLACSO donde dirige el posgrado en Educación y Tecnologías. Formadora de docentes universitarios desde hace 15 años. Consultora en Tecnologías y Educación para organismos nacionales e internacionales. Investiga en didáctica, formación docente y sobre inclusión de tecnologías en educación. https://orcid.org/o0o0-0002-5178-7398

E-mail: gisela.schwartzman@hospitalitaliano.org.ar

Carolina Roni. Doctora en Cs. de la Educación (CONICET-UNLP). Investiga y trabaja en formación docente en el IUHIBA, donde además actualmente ejerce el cargo de Subdirectora del Departamento de Educación. Se especializa en didáctica y pedagogía del nivel medio y superior, situada en las ciencias naturales y las ciencias de la salud. También es investigadora del GICEOLEM con quienes indaga sobre la función epistémica de la escritura y la alfabetización académica. https://orcid. org/0000-0002-3115-3689

E-mail: carolina.roni@hospitalitaliano.org.ar

Michelle Berk. Lic. en Ciencias de la Educación por la Universidad de Buenos Aires, Argentina. Investiga y trabaja en el Departamento de Educación del IUHIBA, donde actualmente se dedica al asesoramiento pedagógico a carreras de posgrado, la acreditación de carreras universitarias, y la formación de docentes de nivel superior; temáticas sobre las cuales también investiga. https://orcid.org/0000-0002-0177$\underline{0715}$

E-mail: michelle.berk@hospitalitaliano.org.ar

Erica Delorenzi. Lic. en Ciencias de la Educación por la Universidad de Buenos Aires. Sub-Coordinadora de Formación Docente IUHIBA. Secretaria Académica de la Especialización en Docencia universitaria para profesionales de la salud del IUHIBA y Asesora pedagógica y docente en el Departamento de Educación de dicha universidad. Investiga sobre formación docente en nivel superior. https://orcid. org/0000-0002-1106-0899

E-mail: erica.delorenzi@hospitalitaliano.org.ar 
Mariana Sánchez. Lic. y Prof. en Ciencias de la Educación (UBA, Argentina). Investiga y trabaja en el Departamento de Educación del IUHIBA, donde actualmente se dedica a la formación de docentes universitarios y a la asesoría pedagógica de carreras de grado. Investiga sobre género y diversidad en la universidad y sobre temas de didáctica vinculados a la formación de docentes universitarios. https:// orcid.org/0000-0003-0316-8602

E-mail: mariana.sanchez@hospitalitaliano.org.ar

María Laura Eder. Doctora en Educación (UBA, Argentina), Magister en Didáctica y Lic. en Ciencias de la Educación (UBA, Argentina). Secretaría académica del Instituto Universitario del Hospital Italiano y de la Maestría en educación para profesionales de la salud, Subdirectora de la especialización en docencia universitaria de la misma universidad. Es formadora de docentes universitarios desde hace más de 25 años. Investiga en temas de didáctica universitaria y sobre la formación docente en la educación superior. https://orcid.org/0000-0002-3941-6834

E-mail: laura.eder@hospitalitaliano.org.ar

\section{DIRECCIÓN DE LOS AUTORES}

Departamento de Educación

Instituto Universitario Hospital Italiano de Buenos Aires (IUHIBA)

Potosí 4234 (1199) - Ciudad de Buenos Aires (Argentina)

Fecha de recepción del artículo: 11/12/2020

Fecha de aceptación del artículo: 15/02/2021

Fecha de aprobación para maquetación: 26/02/2021 